\title{
Development of an in-situ calibration device of firn properties for Askaryan neutrino detectors
}

Jakob Beise ${ }^{a, *}$ and Christian Glaser $^{a}$ on behalf of the ARIANNA Collaboration (a complete list of authors can be found at the end of the proceedings)

${ }^{a}$ Uppsala University, Department of Physics and Astronomy, Box 516, 75120 Uppsala, Sweden

E-mail: jakob.beise@physics.uu.se, christian.glaser@physics.uu.se

High energy neutrinos $\left(E>10^{17} \mathrm{eV}\right)$ are detected cost-efficiently via the Askaryan effect in ice, where a particle cascade induced by the neutrino interaction produces coherent radio emission that can be picked up by antennas installed below the surface. A good knowledge of the near surface ice (aka firn) properties is required to reconstruct the neutrino properties. In particular, a continuous monitoring of the snow accumulation (which changes the depth of the antennas) and the index-of-refraction profile are crucial for an accurate determination of the neutrino's direction and energy. We present an in-situ calibration system that extends the radio detector station with a radio emitter to continuously monitor the firn properties by measuring time differences of direct and reflected (off the surface) signals (D'n'R). We optimized the station layout in a simulation study and quantified the achievable precision. We present 14 months of data of the ARIANNA detector on the Ross Ice Shelf, Antarctica, where a prototype of this calibration system was successfully used to monitor the snow accumulation with unprecedented precision of $1 \mathrm{~mm}$. We explore and test several algorithms to extract the D'n'R time difference from noisy data (including deep learning). This constitutes an in-situ test of the neutrino vertex distance reconstruction using the D'n'R technique which is needed to determine the neutrino energy.

$37^{\text {th }}$ International Cosmic Ray Conference (ICRC 2021)

July 12th - 23rd, 2021

Online - Berlin, Germany

\footnotetext{
${ }^{*}$ Presenter
} 


\section{Introduction}

High-energy (HE) neutrinos constitute a smoking gun signature for the production sites of HE cosmic rays (CR) as they directly pinpoint to their origin giving insights into the most violent processes known to happen in our Universe. Detecting ultra high-energy (UHE) neutrinos with energies above $10^{17} \mathrm{eV}$ is of particular interest as they allow to study the production mechanisms behind the most energetic particles in our Universe.

The most cost-efficient way of covering large effective areas is provided by the radio detection technique based on the Askaryan effect, as the attenuation length of radio signals in cold ice is $O(1 \mathrm{~km})$. The radio emission is a result of a moving, time-varying charge excess during the propagation of the charged secondaries of a shower. Askaryan emission is emitted in the frequency range between $50 \mathrm{MHz}$ to $1 \mathrm{GHz}$ on a narrow cone with the emission being strongest close the the Cherenkov angle.

Radio neutrino detectors are composed of an array of autonomous and individual stations spaced over large distances and maximised for effective area. To this day there are two existing pilot projects ARIANNA, positioned at the Ross Ice Shelf at Moore's Bay and the South Pole [1], and ARA, located at the South Pole [2]. RNO-G at Summit Station on Greenland is currently under construction [3], a detector with similar sensitivity but complementary sky coverage is proposed for the Ross Ice shelf [4] and an order of magnitude larger radio detector is part of the plans for IceCube-Gen2 [5].

For this study a shallow station design is considered encompassing upwards- and downwardsfacing LPDA antennas in depth of $3 \mathrm{~m}$ and a vertically polarised dipole antenna $15 \mathrm{~m}$ below the surface (Fig. 1). This design is proposed for the ARIANNA-200 experiment. A shallow component is also part of the RNO-G design and foreseen for IceCube-Gen2 [5]. The deep dipole will in the vast majority of the neutrino events record two signals: A direct signal propagating on a curved trajectory and a slightly time-delayed, second pulse reflected off the ice-air interface. The D'n'R signature not only provides a unique way of identifying in-ice radio emission against backgrounds but also provides an estimate of the viewing angle [6] and a precise measurement of the distance to the neutrino interaction vertex [7], as the time difference between direct and reflected signal is inversely proportional to the distance. Thus, the D'n'R time difference is required for the neutrino vertex and neutrino energy reconstruction.

As the density of the near surface ice (upper $O(100 \mathrm{~m}$ ), hereafter referred to as firn) changes from fluffy, loose snow at the surface to compacted ice corresponding to a gradient in the indexof-refraction profile, the propagation paths for radio signals arriving at a shallow station are bent downwards. Therefore, to achieve best possible energy reconstruction, the firn properties, including the snow accumulation and the index-of-refraction profile, have to be determined with high accuracy and monitored continuously (in case of snow accumulation) to take temporal changes into account. A prototype calibration system for the snow accumulation was already deployed in the Austral season of 2019 [7] and is successfully running ever since. This paper presents results for the first 14 months of data taking, and thus extends the previously published analysis by a year. In addition, we explore an improved calibration setup consisting of two transmitters instead of just one to include a reconstruction of the index-of-refraction profile. In a simulation study, the position of the two transmitters is optimized, the achievable reconstruction resolution of the firn properties are 


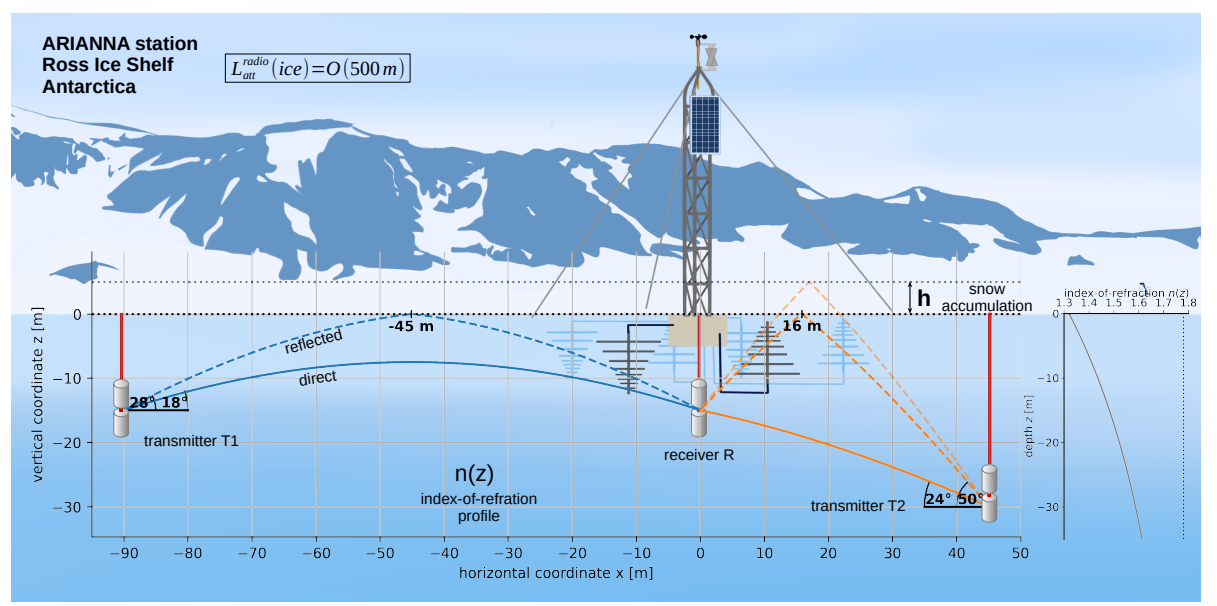

Figure 1: Sketch of a calibration device consisting of two transmitters (T1 and T2) and one receiver (R) antenna. A $15 \mathrm{~m}$ deep dipole picks up the D'n'R signal composed of the early direct signal (solid line) and a slightly time-delayed, second pulse reflected off the ice-air interface (dashed line). The time delay between both signals relates to the snow accumulation. The layout reflects the optimised antenna configuration found in the optimisation study in Sec. 3 which sets T1 to [ $-90 \mathrm{~m},-15 \mathrm{~m}]$ and T2 to [ $-45 \mathrm{~m},-30 \mathrm{~m}]$. In addition, it is exemplified how a change in the snow height $h$ affects the D'n'R time difference by prolonging the reflected path. Also shown are variations in the index-of-refraction profile $n(z)$ which also affect the signal propagation.

determined and systematic uncertainties arising from displacements in deployment are quantified.

\section{In-situ calibration device for the measurement of the snow accumulation}

The snow accumulation calibration device was installed in 2019 [7] as a supplement to station 52 of the ARIANNA radio array at Moore's Bay and comprises a $52 \mathrm{~cm}$ long, $8 \mathrm{~cm}$ diameter receiving dipole antenna in $8.6 \mathrm{~m}$ depth and a $36 \mathrm{~cm}$ long, $5 \mathrm{~cm}$ diameter, copper fat-dipole deployed $42 \mathrm{~m}$ away and $18.2 \mathrm{~m}$ deep in the firn. The emitter antenna is triggered every 12 hours and releases a burst of 150 individual pulses with a repetition rate of $0.5 \mathrm{~Hz}$. In order to increase the signal-tonoise ratio, the 150 single events are averaged. For the extraction of the D'n'R time difference a template of the direct pulse is created. To find the position of the reflected pulse the template is first phase-shifted and attenuated according to the Fresnel equations and then shifted over the signal trace. A peak in the cross correlation (CC) between the signal trace and the reflected template corresponds to the position of the reflected pulse. Using NuRadioMC [8] for ray tracing, the known detector geometry and index-of-refraction profile, the time differences can be translated into snow accumulation.

Figure 2 shows the measured D'n'R time difference and the corresponding snow accumulation over 14 months between December 2018 to February 2020. Instead of a continuous growth of snow height, the total of $60 \mathrm{~cm}$ has accumulated in discrete jumps which coincide with periods of high trigger rates - an indicator for storms. As can be seen from the scatter of the blue data points, the $\mathrm{CC}$ algorithm constitutes a precise and reliable reconstruction method for high SNR signals with up to $5 \mathrm{ps}$ or $1 \mathrm{~mm}$ resolution. 


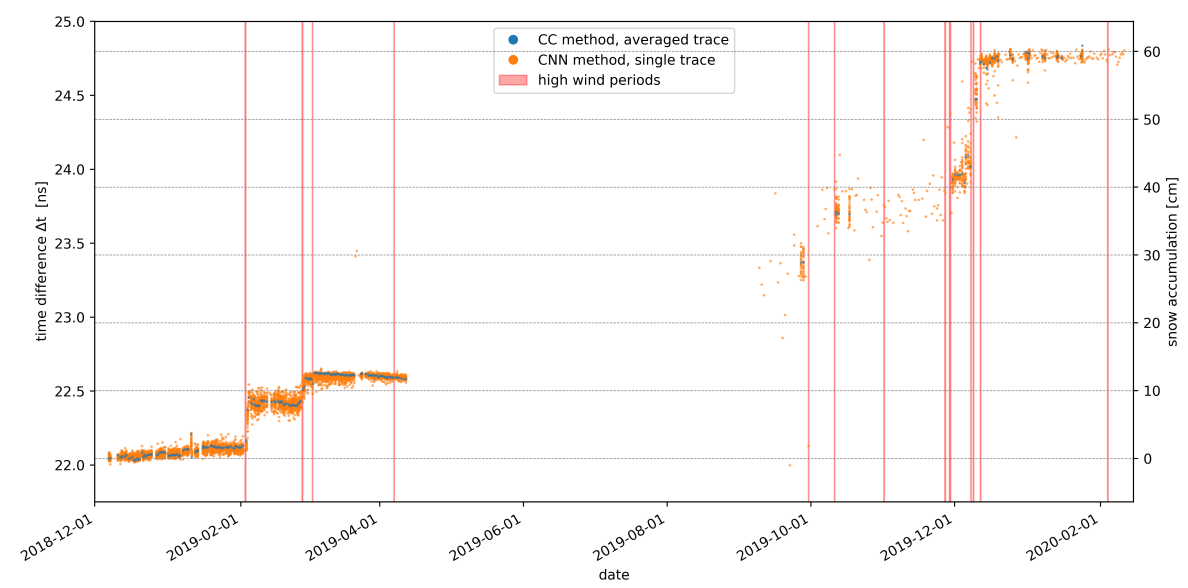

Figure 2: Reconstruction of the D'n'R time difference (left axis) and respectively snow accumulation (right axis) from averaged high-SNR traces with a cross correlation method (blue) and from individual, low-SNR events with a deep learning algorithm (orange). During the dark winter months the station shuts down (April 2019 until September 2020). The red band indicate periods of high wind.

However, it is found that the CC method applied on single, low-SNR traces is less precise with a $1.2 \mathrm{~ns}$ resolution and shows a trend of increasing spread for progressing dates. Therefore, a convolutional neural network (CNN) was trained to reconstruct the D'n'R time difference from the measured single traces. The model has about $1.3 \mathrm{M}$ parameters and is composed of 12 onedimensional convolutional layers of reducing sizes grouped in three blocks of fours layer each, each supplemented by an average pooling layer and completed by five densely connected layers of decreasing dimension. We used $85 \%$ of the data for training and validation where the true time difference was set to the result of the $\mathrm{CC}$ method on the averaged traces. The $\mathrm{CNN}$ reconstruction on low SNR traces (of the test data set that were not used during training or validation plus additional events where only a single event from a burst was transferred via the satellite backup communication link) is shown as orange data points in Fig. 2. The CNN achieves a resolution of 34 ps or $8 \mathrm{~mm}$, which is a reduction of more than a factor 30 compared to the CC method applied on individual events, while not showing any temporal trend. Thus, it can be concluded that the deep learning algorithm can be applied in low-SNR situation for which a traditional CC analysis is less precise. In addition, the single trace reconstruction allows precise reconstruction whenever the data is incomplete as is the case in November 2019 and February 2020.

\section{In-situ calibration for the measurement of firn properties}

Intended as an extension to the snow accumulation device at Moore's Bay to allow for the reconstruction of the index-of-refraction profile, the calibration device is complemented by an additional transmitter antenna. The change of the index-of-refraction with depth $z$ can be described well with a simple exponential function of the form

$$
n(z)=1.78-\Delta n \cdot e^{-z / z_{0}}
$$


where 1.78 is the index-of-refraction of deep ice. The two free parameters $\Delta n$ (the change of refractive index) and $z_{0}$ (the characteristic length) are constraint from measurements. For the Moore's Bay site the parameters are determined from density measurements of borehole ice to $\Delta n=0.46 \pm 0.01$ and $z_{0}=(34.5 \pm 2) \mathrm{m}$ [9]. This implicates that the index-of-refraction changes from 1.32 at the surface for the loose snow to about 1.78 in $200 \mathrm{~m}$ depth. The goal of the proposed calibration system is to precisely determine $\Delta n$ and $z_{0}$.

The calibration system includes two transmitters (T1 and T2) and a receiver antenna (R). Each transmitter will generate a direct and reflected signal pulse in the receiver which correspond to three independent time delay measurements. This allows to determine the snow accumulation $h$ and the two parameters $\Delta n$ and $z_{0}$ of the $n(z)$ profile simultaneously. The receiver is placed at a depth of $15 \mathrm{~m}$ which is optimal for the neutrino energy reconstruction [7]. Because the index-ofrefraction only depends on depth and due to the radial symmetry of the setup, the further study is reduced to two spacial dimensions and only the horizontal distance and depth of the emitters need to be optimized. The positions of the two emitters is optimized to give the highest reconstruction resolution on $h, \Delta n$ and $z_{0}$ while also minimising correlation between the parameters. The allowed antenna space is restrained by the 'shadow zone', as signals from emitter placed therein will not reach the receiver. Furthermore, a high enough signal strengths of the reflected signal is required to enable efficient reconstruction, which further constrains possible positions to those exceeding $90 \%$ reflectivity in the ice-air reflection.

We test all possible combinations of emitter positions on a grid with a spacing of $5 \mathrm{~m}$ in $x$ and $z$, spanning depths from $-10 \mathrm{~m}$ to $-40 \mathrm{~m}$ and an horizontal distances from $-10 \mathrm{~m}$ to $-150 \mathrm{~m}$. The depths range is a compromise between drilling efforts and reconstruction performance, while the $x$-range covers all positions to the 'shadow zone' (cf. Fig. 3). The grid allows for $125 \times 124=15500$ possible combinations of transmitter antennas, however only half of them, 7750, are distinct as the interchange of the identical antennas $\mathrm{T} 1$ and $\mathrm{T} 2(\mathrm{~T} 1 \rightarrow \mathrm{T} 2, \mathrm{~T} 2 \rightarrow \mathrm{T} 1)$ is just a change of labels and does not affect the underlying physics.

For each of the configurations, NuRadioMC's ray tracer is utilised to solve for the four propagation times (direct and reflected signal for both transmitters). For the simultaneous reconstruction of $h, \Delta n$ and $z_{0}$, at least three observables are required, which are constructed as the relative time differences between the propagation time of the direct pulse from $\mathrm{T} 1$ to the remaining three propagation times. In order to simulate a real measurement with statistical uncertainties, the propagation times are smeared 10,000 times according to a Gaussian distribution with a timing resolution of $0.2 \mathrm{~ns}$.

We estimate the achievable resolution for each emitter pair by performing a least-square optimization of the firn properties $h, \Delta n$ and $z_{0}$. For each pair of emitter positions, 200 different data sets consisting of 10,000 individual measurements each are generated as described above. We then, for each data set, determine the most probably values of the firn properties $h, \Delta n$ and $z_{0}$ by performing a least-square minimization. This is done in order to estimate the reconstruction resolution and the correlation from the parameter distribution for the parameters of the firn properties. As the optimal configuration is a compromise in reconstruction performance for the three different parameters it is useful to define a variable that combines the reconstruction resolution and the correlation in $h, \Delta n$ and $z_{0}$. This is incorporated in the combined reconstruction resolution $\sigma_{c o m b}$ and the combined correlation $\rho_{c o m b}$ which are the sum of the squares of the individual contributions normalised to 

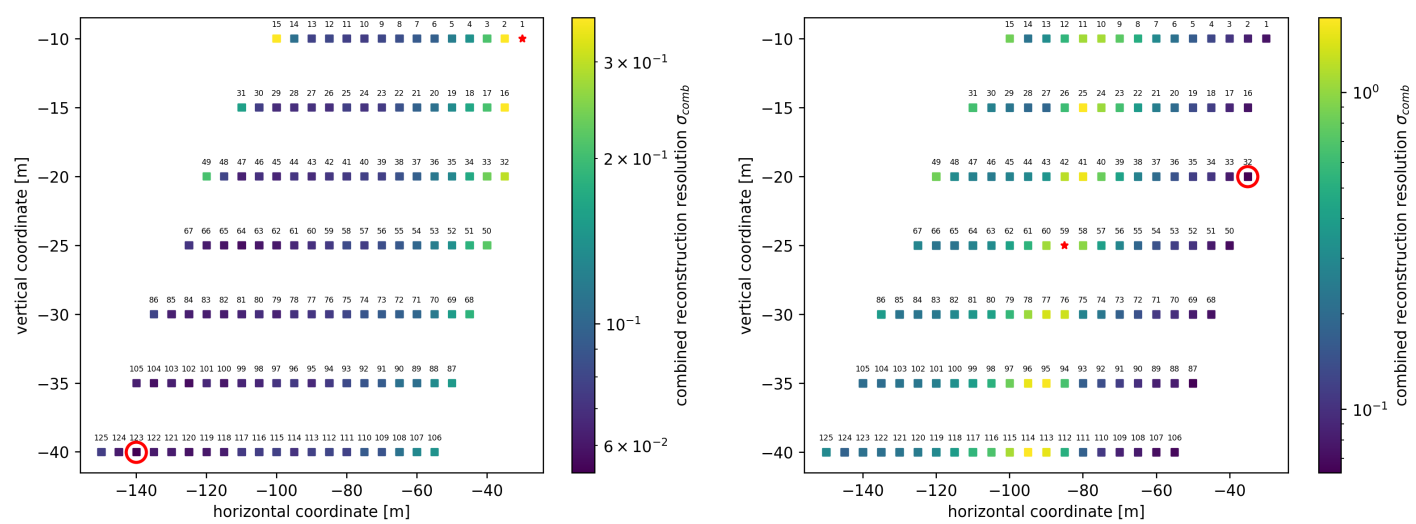

Figure 3: For a fixed position of $\mathrm{T} 1$ (red star) there is a position of $\mathrm{T} 2$ that yields minimal reconstruction resolution (red circle). Left: T1 in the upper right at position 1; optimal T2 at position 123. Right: T1 in the middle at position 59, optimal $\mathrm{T} 2$ at position 32 . The pairs $\mathrm{T} 1-\mathrm{T} 2$ are maximally opposed in space.

a target resolution to account for the different orders of magnitude in the spread of the parameter distribution. The normalisation was chosen to be $1 \%$ for the parameters of the $n(z)$-profile and $1 \mathrm{~cm}$ in snow accumulation, which is a pessimistic benchmark for what the calibration system should achieve.

$$
\begin{aligned}
& \sigma_{\text {comb }}=\sqrt{\left(\sigma_{h} / 1 \mathrm{~cm}\right)^{2}+\left(\sigma_{\Delta n} / 0.0046\right)^{2}+\left(\sigma_{z_{0}} / 34.5 \mathrm{~cm}\right)^{2}} \\
& \rho_{c o m b}=\sqrt{\rho_{h, \Delta n}^{2}+\rho_{h, z_{0}}^{2}+\rho_{\Delta n, z_{0}}^{2}} .
\end{aligned}
$$

Figure 3 depicts T1 placed at specific position (red star) indicated by the upper index (left figure: T1 at position 1 - upper right, right figure: T1 at position 59 - middle) on the grid, while the coloured squares indicate the combined reconstruction resolution for T2 placed at the respective position. The red circled square marks the position of $\mathrm{T} 2$ where the reconstruction resolution is minimal for a corresponding T1. The plots demonstrate, that if the positions of both transmitters are extremely opposed, best results in the combined reconstruction resolution are obtained. This can be easily understood when considering the extreme case where both transmitters are placed at the same position, leading to four, degenerate trajectories.

For each possible position of transmitter T1 we can now calculate the optimal position of emitter T2 which we show in Fig. 4. Among all T1-T2-pairs there is a combination that achieves the best reconstruction resolution (cf.Fig. 4 (left)). However, that configuration may result in a large correlation between the parameters. Because we prefer a slightly worse reconstruction resolution with less correlation between the parameters, we calculate the combined correlation (Eq. (3)) the subset of emitter pairs with a combined resolution within $20 \%$ of the best achievable resolution. Figure 4 (right) shows the result. The position that has the minimal combined correlation while worsening the reconstruction resolution by less than $20 \%$ is found to be $27-68$ at position [ $-90 \mathrm{~m},-15 \mathrm{~m}]$ and $[-45 \mathrm{~m},-30 \mathrm{~m}]$. The achievable reconstruction resolution and correlation in $h$, $\Delta n$ and $z_{0}$ for the reconstruction resolution-optimised of configuration and the correlation-optimised configuration are listed in Tab. 1. For the correlation-minimised layout, the propagation paths are 

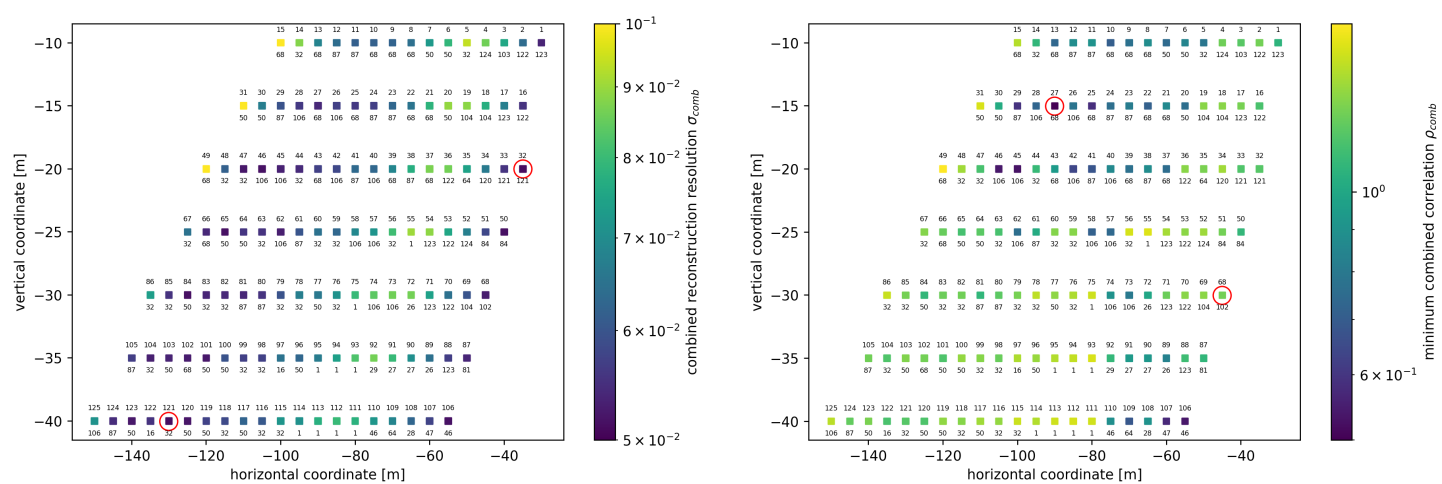

Figure 4: For every possible position of emitter T1 (numbered by the upper index), the figures show the corresponding, optimal position of emitter T2 (numbered by the lower index) and the obtainable resolution as color code. The best pair is indicated with red circles. (left) combined reconstruction resolution and (right) and combined correlation.

\begin{tabular}{cccc}
\hline \hline \multicolumn{4}{c}{ Reconstruction resolution-optimised configuration } \\
\hline reconstruction resolution & $\sigma_{h}=5 \mathrm{~mm}$ & $\sigma_{\Delta n} / \Delta n=0.05 \%$ & $\sigma_{z_{0}} / z_{0}=0.1 \%$ \\
correlation & $\rho_{h, \Delta n}=60 \%$ & $\rho_{h, z_{0}}=-53 \%$ & $\rho_{\Delta n, z_{0}}=-87 \%$ \\
\hline \hline \multicolumn{5}{c}{ Correlation-optimised configuration } \\
\hline reconstruction resolution & $\sigma_{h}=5 \mathrm{~mm}$ & $\sigma_{\Delta n} / \Delta n=0.04 \%$ & $\sigma_{z_{0}} / z_{0}=0.3 \%$ \\
correlation & $\rho_{h, \Delta n}=24 \%$ & $\rho_{h, z_{0}}=-41 \%$ & $\rho_{\Delta n, z_{0}}=-12 \%$ \\
\hline \hline
\end{tabular}

Table 1: Per-100-events reconstruction resolution and correlation for the correlation-optimised pair in Fig. 4 (right).

depicted in Fig. 1 as well as the point-of-reflection and the angle of the launch vector.

We rescaled the statistical uncertainty from 10,000 events per measurement to just 100 events which increases the uncertainty by a factor of $10(=\sqrt{100})$. We find a statistical uncertainty of $5 \mathrm{~mm}$ in $h$ and $0.04 \%$ and $0.3 \%$ in $\Delta n$ and $z_{0}$ respectively, which poses an improvement of a factor of 50 and 20 than previous measurements from borehole measurements of the density profile for $\Delta n$ and $z_{0}$. And maybe more importantly, the measurement is directly based on the propagation times of radio signals in ice and not on an empirical correlation between density and index-of-refraction. According the previous studies [1], an uncertainty in the snow accumulation $h$ of $5 \mathrm{~mm}$ is about a factor of 10 lower than the required resolution needed for a precise vertex reconstruction. The correlation coefficient ranges between $-41 \%$ to $24 \%$ indicating that there is considerable correlation between the firn parameters which does not come at a surprise, as variations in the snow accumulation are closely linked to changes in the index-of-refraction profile. In addition to a preciser neutrino energy reconstruction by a better knowledge of the $n(z)$-profile, potential temporal variations of the index-of-refraction profile can be monitored in real-time.

We also investigated the impact of uncertainties in the emitter and receiver positions and found that an systematic uncertainty in the position of $1 \mathrm{~cm}$ results in systematic uncertainty of $0.05 \%$ in $\Delta n$ and $0.12 \%$ in $z_{0}$ which is respectively about $25 \%$ larger or a factor of 3 smaller than the statistical error. The systematic uncertainty of $\Delta n$ and $z_{0}$ scales roughly linear with the uncertainty in the emitter/receiver position. Thus, in order to keep the systematic uncertainty below the statistical, 
one needs to locate the antennas in $x$ and $z$ with a resolution of $\pm 1 \mathrm{~cm}$.

\section{Conclusions}

The D'n'R technique provides unique opportunities for the detection of high-energy neutrinos. An antenna placed $O(15 \mathrm{~m})$ below the ice surface will measure a direct pulse and a pulse reflected off the ice surface for most detected neutrino interactions in the ice. This signature does not only provide a unique characteristic of a neutrino origin of the signal but also allows to measure viewing angle as well as the distance to the neutrino interaction vertex precisely, a crucial property to determine the neutrino energy and direction. The D'n'R technique was tested experimentally at Moore's Bay on the Ross ice shelf. An ARIANNA detector station equipped with a $8.6 \mathrm{~m}$ deep dipole antenna received signals from a $20 \mathrm{~m}$ deep transmitter. This setup was used to continuously monitor the snow accumulation which is proportional to the time delay between the direct and reflected pulse. We have presented 14 months of data where we extracted the time delays via cross correlation and deep learning algorithms, reaching unprecedented precision of $O(1 \mathrm{~mm})$ in snow accumulation.

We simulated a possible extension of the calibration setup by adding a second emitter to also determine the depth-dependence of the index-of-refraction profile. We determined the optimal positions of two emitters and found that this setup can outperform current density-based measurements of the index-of-refraction profile by an order of magnitude. The setup even allows for a continuous monitoring of the firn properties. Equipping a few radio detector stations of a future array will be beneficial to confirm uniform and stable firn properties.

\section{Acknowledgements}

We are grateful to the U.S. National Science Foundation-Office of Polar Programs, the U.S. National Science Foundation-Physics Division (grant NSF-1607719) for supporting the ARIANNA array at Moore's Bay. Without the invaluable contributions of the people at McMurdo Station, the ARIANNA stations would have never been built. We acknowledge funding from the German research foundation (DFG) under grants GL 914/1-1 and NE 2031/2-1m, the Taiwan Ministry of Science and Technology, the Swedish Government strategic program Stand Up for Energy, MEPhI Academic Excellence Project (Contract No. 02.a03.21.0005) and the Megagrant 2013 program of Russia, via agreement 14.12.31.0006 from 24.06.2013. This work was supported by National Science Foundation Grant DGE-1633631.

\section{References}

[1] ARIANNA Collaboration, A. Anker et al. JCAP 03 (2020) 053.

[2] ARA Collaboration, P. Allison et al. Phys. Rev. D 102 (2020) 043021.

[3] RNO-G Collaboration, J. A. Aguilar et al. JINST 16 P03025 2021.

[4] S. Barwick for the ARIANNA collaboration PoS ICRC2021 (these proceedings) xxx.

[5] S. Hallmann for the IceCube-Gen2 collaboration PoS ICRC2021 (these proceedings) xxx.

[6] S. Barwick for the ARIANNA collaboration PoS ICRC2021 (these proceedings) xxx.

[7] ARIANNA Collaboration, A. Anker et al. JCAP 11 (2019) 030.

[8] C. Glaser et al. EPJ C80 no. 2, (2020) 77.

[9] S. W. Barwick et al. JCAP07(2018)055. 


\section{Full Authors List: ARIANNA Collaboration}

Astrid Anker ${ }^{1}$, Pierre Baldi ${ }^{2}$, Steven W. Barwick ${ }^{1}$, Jakob Beise ${ }^{3}$, Hans Bernhoff ${ }^{4}$, Dave Z. Besson ${ }^{5,6}$, Nils Bingefors ${ }^{3}$, Maddalena Cataldo $^{7}$, Pisin Chen ${ }^{8}$, Daniel García Fernández ${ }^{9,7}$, Geoffrey Gaswint $^{1}$, Christian Glaser ${ }^{3}$, Allan Hallgren ${ }^{3}$, Steffen Hallmann ${ }^{9}$, Jordan C. Hanson ${ }^{10}$, Spencer R. Klein ${ }^{11}$, Stuart A. Kleinfelder ${ }^{12}$, Robert Lahmann ${ }^{7,1}$, Jiayi Liu ${ }^{1}$, Mitchell Magnuson ${ }^{5}$, Stephen McAleer ${ }^{2}$, Zach Meyers ${ }^{9}$, Jiwoo $\mathrm{Nam}^{8}$, Anna Nelles ${ }^{9,7}$, Alexander Novikov ${ }^{5,6}$, Manuel P. Paul ${ }^{1}$, Christopher Persichilli ${ }^{1}$, Ilse Plaisier ${ }^{9,7}$, Lilly Pyras $^{9}$, Ryan Rice-Smith ${ }^{1}$, Joulien Tatar ${ }^{13}$, Shih-Hao Wang ${ }^{8}$, Christoph Welling ${ }^{9,7}$, Leshan Zhao ${ }^{1}$

${ }^{1}$ Department of Physics and Astronomy, University of California, Irvine, CA 92697, USA.

${ }^{2}$ Department of Information and Computer Science, University of California, Irvine, CA 92697, USA.

${ }^{3}$ Uppsala University Department of Physics and Astronomy, Uppsala SE-752 37, Sweden.

${ }^{4}$ Uppsala University Department of Engineering Sciences, Division of Electricity, Uppsala SE-752 37, Sweden.

${ }^{5}$ Department of Physics and Astronomy, University of Kansas, Lawrence, KS 66045, USA.

${ }^{6}$ National Research Nuclear University MEPhI (Moscow Engineering Physics Institute), Moscow 115409, Russia.

${ }^{7}$ ECAP, Friedrich-Alexander Universität Erlangen-Nürnberg, 91058 Erlangen, Germany.

${ }^{8}$ Department of Physics and Leung Center for Cosmology and Particle Astrophysics, National Taiwan University, Taipei 10617, Taiwan. ${ }^{9}$ DESY, 15738 Zeuthen, Germany.

${ }^{10}$ Whittier College Department of Physics, Whittier, CA 90602, USA.

${ }^{11}$ Lawrence Berkeley National Laboratory, Berkeley, CA 94720, USA.

${ }^{12}$ Department of Electrical Engineering and Computer Science, University of California, Irvine, CA 92697, USA.

${ }^{13}$ Research Cyberinfrastructure Center, University of California, Irvine, CA 92697, USA. 